\title{
NMR Observation of Rattling Phonons in the Pyrochlore Superconductor $\mathrm{KO}_{2} \mathrm{O}_{6}$
}

\author{
M. Yoshida, K. Arai, R. Kaido, M. Takigawa, S. Yonezawa, Y. Muraoka, and Z. Hiroi \\ Institute for Solid State Physics, University of Tokyo, Kashiwanoha, Kashiwa, Chiba 277-8581, Japan
}

(Dated: October 20, 2018)

\begin{abstract}
We report nuclear magnetic resonance studies on the $\beta$-pyrochlore oxide superconductor $\mathrm{KO}_{2} \mathrm{O}_{6}$. The nuclear relaxation at the $\mathrm{K}$ sites is entirely caused by fluctuations of electric field gradient, which we ascribe to highly anharmonic low frequency oscillation (rattling) of K ions. A phenomenological analysis shows a crossover from overdamped to underdamped behavior of the rattling phonons with decreasing temperature and its sudden sharpening below the superconducting transition temperature $T_{c}$. Suppression of the Hebel-Slichter peak in the relaxation rate at the $\mathrm{O}$ sites below $T_{c}$ also indicates strong electron-phonon coupling.
\end{abstract}

PACS numbers: 76.60.-k, 74.70.-b, 74.25.Kc

The family of new superconductors $A \mathrm{Os}_{2} \mathrm{O}_{6}(A=\mathrm{K}$, $\mathrm{Rb}$, and $\mathrm{Cs}$ ) with the pyrochlore structure exhibits many exotic properties. For instance, the superconducting transition temperature $T_{c}$ increases substantially with decreasing radius of $A$ ions $\left(T_{c}=3.3,6.3\right.$, and $9.6 \mathrm{~K}$ for $A=\mathrm{Cs}, \mathrm{Rb}$, and $\mathrm{K}$, respectively $[1,2,3,3,4])$ and shows non-monotonic pressure dependence [5], in contradiction to what would be expected from the change of density of states alone. Among this family $\mathrm{KOs}_{2} \mathrm{O}_{6}$ is most anomalous. The electrical resistivity shows strong concave $T$ dependence down to $T_{c}$ [1, 6], in contrast to the normal $T^{2}$-behavior in $\mathrm{Rb}$ and $\mathrm{Cs}$ compounds $[2,3,4,4]$. The $T$ linear coefficient of the specific heat $\gamma=70 \mathrm{~mJ} / \mathrm{K}^{2} \mathrm{~mol}$ [7, 8] is highly enhanced over the value obtained from band calculations $10-11 \mathrm{~mJ} / \mathrm{K}^{2} \mathrm{~mol}$ [9, 10].

These anomalies imply large density of low energy excitations, which was originally ascribed to magnetic frustration inherent to the pyrochlore lattice, a network of corner-shared tetrahedra formed by Os atoms. However, the Pauli-like susceptibility [7] excludes local moments on the Os sites. How the frustrated lattice geometry affects properties of itinerant electrons is still an open issue. On the other hand, there is now a growing body of experimental evidence for anharmonic motion of isolated alkaline ions in an oversized cage of Os-O network. The specific heat data show existence of low frequency Einstein modes in addition to the electronic and the Debye contributions for all members of $A \mathrm{Os}_{2} \mathrm{O}_{6}$ [7, $\left., 8,11\right]$. The $\mathrm{X}$-ray results revealed huge atomic displacement parameter for the $\mathrm{K}$ sites in $\mathrm{KOs}_{2} \mathrm{O}_{6}$ [12]. Recently a second phase transition at $T_{p}=7.5 \mathrm{~K}$ was observed for high quality single crystals of $\mathrm{KOs}_{2} \mathrm{O}_{6}[6]$. Since the transition is of first order and persists into the normal state in high magnetic fields, it is likely a structural transition associated with the $\mathrm{K}$ ion dynamics. Calculation of effective ionic Hamiltonian also indicates instability of $\mathrm{K}$ ions in a highly anharmonic potential [13], which might be the origin of various anomalies. Oscillations of isolated ions in a large space have been discussed also in other materials such as clathrate [14] and skutterudite [15] compounds and commonly called "rattling".
In this Letter, we report results of nuclear magnetic resonance (NMR) experiments on the ${ }^{39,41} \mathrm{~K}$ nuclei $(\mathrm{nu}-$ clear spin $I=3 / 2)$ and ${ }^{17} \mathrm{O}$ nuclei $(I=5 / 2)$ in $\mathrm{KOs}_{2} \mathrm{O}_{6}$. We found that the nuclear relaxation at the $\mathrm{K}$ sites is caused entirely by phonons via the electric quadrupole interaction. From a phenomenological analysis we propose that rattling phonons undergo a crossover from underdamped to overdamped behavior with increasing temperature. Strong electron-phonon coupling is indicated by rapid reduction of the damping below $T_{c}$. On the other hand, the relaxation rate at the $\mathrm{O}$ sites is sensitive to electronic excitations. Substantial damping of quasiparticles due to rattling phonons near $T_{c}$ is indicated by suppression of the Hebel-Slichter peak at the $\mathrm{O}$ sites. A part of the ${ }^{39} \mathrm{~K}$ NMR results has been discussed in terms of spin fluctuations in a previous publication [16], which must now be discarded.

Two powder samples of $\mathrm{KOs}_{2} \mathrm{O}_{6}$ were used in the present experiment. The sample A was prepared by heating a mixture of $\mathrm{KO}_{2}$ and $\mathrm{OsO}_{2}$ with $\mathrm{Ag}_{2} \mathrm{O}$ as an oxidizing agency [1]. The sample $\mathrm{B}$ was enriched with ${ }^{17} \mathrm{O}$ by heating $\mathrm{KO}_{2}$ and Os metal in two steps in oxygen atmosphere containing $45 \%{ }^{17} \mathrm{O}$. Both samples show identical $T_{c}$ of $9.6 \mathrm{~K}$. Note that the second transition at $7.5 \mathrm{~K}$ has never been observed in powder samples. The nuclear spin-lattice relaxation rate $1 / T_{1}$ at the ${ }^{39,41} \mathrm{~K}$ sites was measured by the saturation recovery method. Although the $\mathrm{K}$ sites have tetrahedral $\left(\mathrm{T}_{d}\right)$ symmetry, hence should not suffer quadrupole broadening, the ${ }^{39} \mathrm{~K}$ NMR spectra consist of a narrow central line $(0.3 \mathrm{kHz}$ HWHM at $8.5 \mathrm{~T})$ and a slightly broad satellite line $(10 \mathrm{kHz}$ HWHM $)$ probably due to imperfections. The line shape shows no appreciable temperature $(T)$ dependence above $T_{c}$. The entire spectrum was easily saturated by rf comb-pulses, resulting in single exponential recovery of the spin echo intensity. The ${ }^{17} \mathrm{O}$ NMR spectra show a quadrupole broadened powder pattern. To determine $1 / T_{1}$, the recovery of the spin-echo intensity of the central line as a function of time $t$ after the inversion pulse was fit to the form [17] $M_{e q}-M_{0}\left\{U \exp \left(-t / T_{1}\right)+V \exp \left(-6 t / T_{1}\right)+(1-U-\right.$ $\left.V) \exp \left(-15 t / T_{1}\right)\right\}$ with $U$ and $V$ fixed to the same values 
at all temperatures.

The $T$-dependences of $1 /\left(T_{1} T\right)$ at the ${ }^{39} \mathrm{~K}$ sites is shown in Fig. 1. The inset of Fig. 1(a) displays the isotopic ratio ${ }^{39} T_{1} /{ }^{41} T_{1}$ for the sample A. Surprisingly, the isotopic ratio coincides with the squared ratio of the nuclear quadrupole moments $\left({ }^{41} Q /{ }^{39} Q\right)^{2}=1.48$ rather than the nuclear magnetic moments $\left({ }^{41} \gamma /{ }^{39} \gamma\right)^{2}=0.30$ in a wide $T$-range $6-100 \mathrm{~K}$. Thus the relaxation is entirely caused by fluctuations of electric field gradient (EFG) as opposed to magnetic fluctuations. Since any active electronic states should be dominantly $s$-like at the K sites with negligibly small quadrupole coupling to K nuclei, it must be phonons that causes the nuclear relaxation.

The prominent features of the data in Fig. 1 are: (1) a peak near $13 \mathrm{~K}(16 \mathrm{~K})$ for the sample B (A), (2) approximate constant behavior at high temperatures, (3) rapid decrease at low temperatures, (4) a clear kink at the superconducting $T_{c}$ and sudden decrease below $T_{c}$. The last point indicates strong influence of superconductivity on the phonon dynamics, a direct evidence for strong
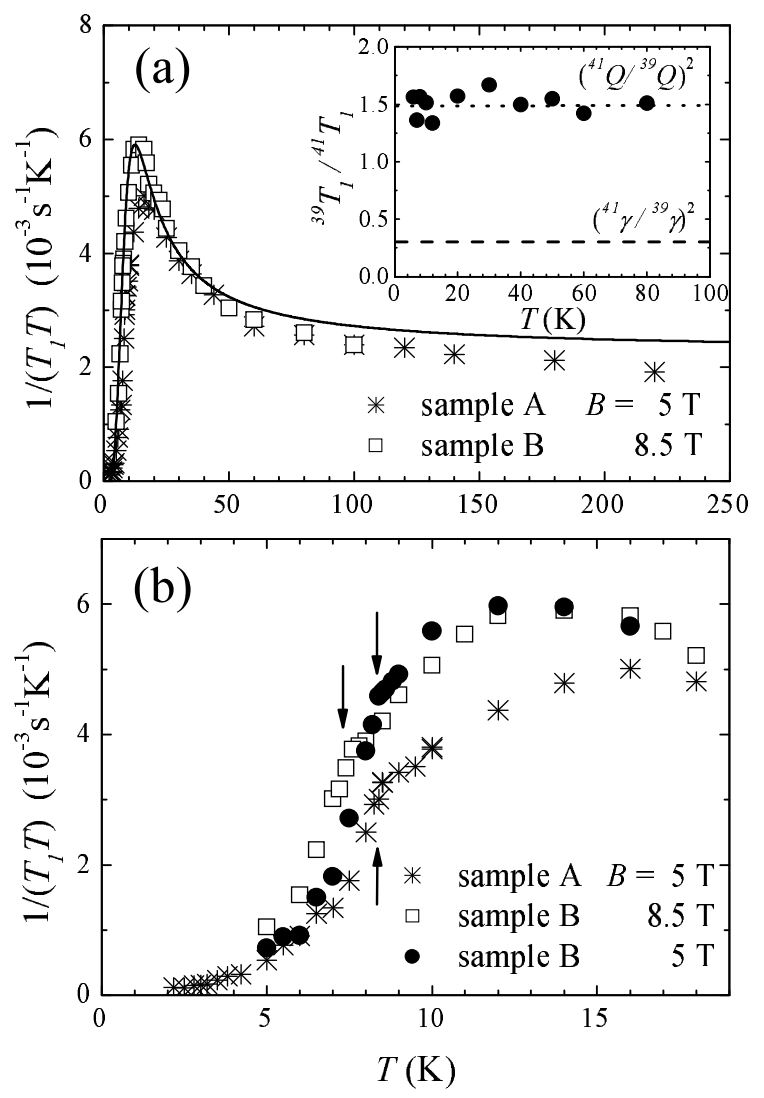

FIG. 1: $T$-dependence of $1 /\left(T_{1} T\right)$ at the ${ }^{39} \mathrm{~K}$ sites for the two samples at different magnetic fields. The data for a wide $T$ range are shown in (a). The solid line shows the calculated result of Eq. (5) as described in the text. The inset shows the isotopic ratio of $1 / T_{1}$. The dotted (dashed) line indicates the squared ratio of the nuclear quadrupole (magnetic) moments. The data in the low $T$ region are expanded in (b). The arrows show the superconducting $T_{c}$ at the respective fields. electron-phonon coupling. The two samples show slightly different results near the peak temperature. Although the reason is not understood clearly, this may arise from different degrees of imperfection or hydration 7].

For nuclei with spin $3 / 2$, the transition probability $W_{q}$ between two nuclear levels $\left|I_{z}=m\right\rangle$ and $\left|I_{z}=m \pm q\right\rangle$ ( $\mathrm{q}=1$ or 2 ) due to quadrupole coupling is given by the correlation function of EFG as [18]

$$
W_{q}=\frac{1}{12}\left(\frac{e Q}{\hbar}\right)^{2} \int_{-\infty}^{\infty}\left\langle\left[V_{+q}(t), V_{-q}(0)\right]\right\rangle e^{i q \omega_{L} t} d t
$$

where $\omega_{L}$ is the nuclear Larmor frequency and $[A, B]$ stands for $(A B+B A) / 2$. The irreducible components of the EFG tensor are defined as $V_{ \pm 1}=V_{x z} \pm i V_{y z}$, $V_{ \pm 2}=\left(V_{x x}-V_{y y} \pm 2 i V_{x y}\right) / 2$, where $V_{x y}=\partial^{2} V / \partial x \partial y$ etc. is the second derivative of the electrostatic potential at the nucleus. For simplicity we assume spherically symmetric EFG fluctuations. Then $W_{1}=W_{2} \equiv W$ and the relaxation rate is given as $1 / T_{1}=2 W$.

In diamagnetic insulators phonons are usually the dominant source of relaxation for quadrupolar nuclei. The ionic motion modulates the EFG,

$$
V_{ \pm q}=V_{ \pm q, 0}+\frac{\partial V_{ \pm q}}{\partial u} u+\frac{1}{2} \frac{\partial^{2} V_{ \pm q}}{\partial u^{2}} u^{2}+\cdots
$$

where $u$ is the ionic displacement from the equilibrium position expressed as a linear combination of the phonon creation $\left(a^{\dagger}\right)$ and annihilation $(a)$ operators. The second term inserted into Eq. (1) leads to the direct process [18], which is expressed by the one phonon correlation function or the imaginary part of the susceptibility,

$$
W_{q} \propto k_{B} T \frac{\operatorname{Im} \chi\left(q \omega_{L}\right)}{q \hbar \omega_{L}}
$$

provided $q \hbar \omega_{L} \ll k_{B} T$. For harmonic phonons with infinite life time, the contribution from the direct process is negligible since $\omega_{L}(\sim 10 \mathrm{MHz})$ is many orders of magnitude smaller than the typical phonon frequency and the phonon density of states at $\omega_{L}$ is practically zero.

On the other hand, the third term in Eq. (2) leads to the two phonon Raman process, in which a nuclear transition occurs by absorbing one phonon and emitting another phonon [18]. Usually this is by far the dominant process expressed as $1 / T_{1} \propto \int\{\rho(\omega)\}^{2} n(\omega)(n(\omega)+$ 1) $|A(\omega)|^{2} d \omega$, where $n(\omega)$ is the Bose factor, $\rho(\omega)$ is the phonon density of states, and $|A(\omega)|$ is the transition matrix element. One can see $1 / T_{1} \propto T^{2}$ at high temperatures where $n(\omega) \sim T / \omega$. At low temperatures, $1 / T_{1}$ decreases monotonically and approaches either to $T^{7}$ dependence for acoustic phonons or activated behavior for optical phonons [18]. Our results in Fig. 1 are, however, in strong contradiction with such behavior.

Since dominance of phonons for nuclear relaxation is extremely rare in metals, we suppose that rattling makes 
a special case for $\mathrm{KOs}_{2} \mathrm{O}_{6}$. The distinct features of rattling are its low energy scale and strong anharmonicity, which should lead to damping. We expect that dampinginduced broadening of the phonon spectrum peaked at a very low frequency, will bring substantial spectral weight at zero frequency so that the direct process (Eq. 3) becomes important. The peak in $1 /\left(T_{1} T\right)$ is then naturally explained as follows. Let us assume a low frequency Einstein mode at $\omega_{0}$. At low temperatures, the spectrum $\operatorname{Im} \chi(\omega) / \omega$ is sharp, therefore, the weight at $\omega_{L} \approx 0$ should be small. As the spectral width increases with increasing temperature, the weight at $\omega=0$ will grow and take a maximum when the width becomes comparable to $\omega_{0}$. Further broadening, however, will spread the spectrum over higher frequencies and reduce the weight at $\omega=0$. (See the inset of Fig. 2.) Thus the peak in $1 /\left(T_{1} T\right)$ can be reproduced if the rattling undergoes a crossover from underdamped to overdamped behavior with increasing temperature. The contribution from the Raman process should be also influenced by damping [19]. However, we could not reproduce the peak in $1 /\left(T_{1} T\right)$ by the Raman process without fine tuning the $T$-dependence of the damping. We consider that the Raman process is unimportant. The details will be discussed elsewhere [20].

In order to quantify the above picture, we employ a suitable phenomenological model describing strongly damped oscillators. A commonly used expression is the damped harmonic oscillator model, $\operatorname{Im} \chi(\omega) / \omega=$ $\Gamma /\left\{\left(\omega_{0}^{2}-\omega^{2}\right)^{2}+\Gamma^{2} \omega^{2}\right\}$, which leads to $1 /\left(T_{1} T\right) \propto \Gamma / \omega_{0}^{4}$ by taking the limit $\omega_{L} \rightarrow 0$ in Eq. (3). However, this model is unable to reproduce the observed peak for any monotonic change of the damping $\Gamma$. In the damped harmonic oscillator model, only the friction or the momentum relaxation is considered as the sources of damping. It was argued, however, that in the collision dominated regime

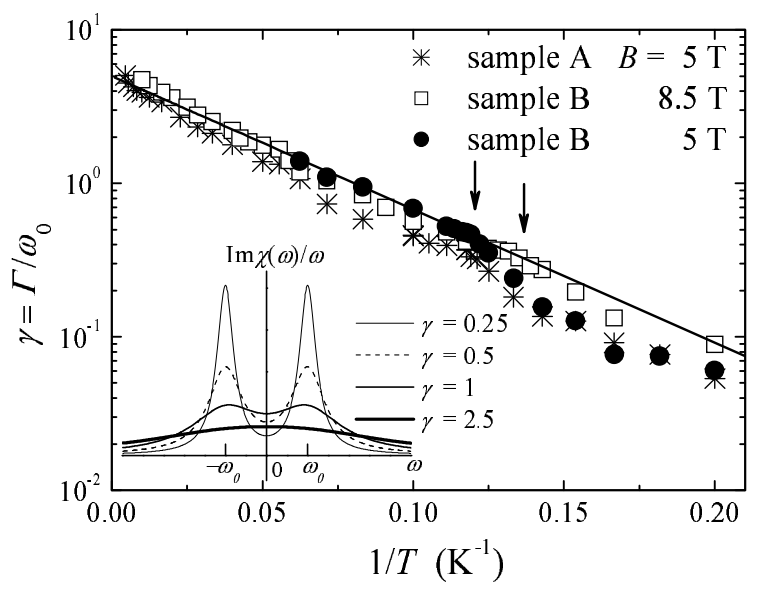

FIG. 2: The normalized damping $\gamma=\Gamma / \omega_{0}$ is plotted against $1 / T$. The arrows correspond to $T_{c}$ at $5 \mathrm{~T}$ and $8.5 \mathrm{~T}$. The inset shows the phonon line shape $\operatorname{Im} \chi(\omega) / \omega$ calculated from Eq. (4) for several values of $\gamma$. one must also consider the jump of the spatial coordinate or the amplitude relaxation [21, 22]. One then obtains the van Vleck-Weisskopf formula [23], which should be suitable for strongly damped anharmonic phonons,

$$
\frac{\operatorname{Im} \chi(\omega)}{\omega}=\frac{\Gamma / \omega_{0}}{\left(\omega+\omega_{0}\right)^{2}+\Gamma^{2}}+\frac{\Gamma / \omega_{0}}{\left(\omega-\omega_{0}\right)^{2}+\Gamma^{2}} .
$$

From Eqs. (4) and (3) we obtain in the limit $\omega_{L} \rightarrow 0$,

$$
\frac{1}{T_{1} T} \propto \frac{1}{\omega_{0}^{2}} \frac{\gamma}{1+\gamma^{2}}
$$

where $\gamma=\Gamma / \omega_{0}$. For a fixed value of $\omega_{0}$, this formula has a maximum at $\gamma=1$, which corresponds to the observed peak. By identifying the $T$-ranges below or above the peak temperature with the underdamped $(\gamma \leq 1)$ or the overdamped $(\gamma \geq 1)$ regions and assuming that $\omega_{0}$ is constant, we extracted the T-dependence of $\gamma$ from the experimental data of $1 /\left(T_{1} T\right)$ as shown in Fig. 2 . The result above $T_{c}$ is well represented by an activation law $\gamma=\gamma_{0} \exp \left(-E / k_{B} T\right)$ with $\gamma_{0}=5$ and $E=20 \mathrm{~K}$ (the solid line). Thus our analysis predicts extremely overdamped behavior at high temperatures. Note that the phonon frequency $\omega_{0}$ cannot be determined from our analysis. The solid line in Fig. 1 shows the $T$-dependence of $1 /\left(T_{1} T\right)$ calculated from Eq. (5) and this activation law. A possible interpretation of the activated behavior is that $\Gamma$ represents the hopping frequency of the $\mathrm{K}$ ions among different metastable positions in highly anharmonic potential [13] and the activation energy corresponds to the potential barrier between them.

We have also measured the relaxation rate for ${ }^{85} \mathrm{Rb}$ and ${ }^{87} \mathrm{Rb}$ nuclei in $\mathrm{RbOs}_{2} \mathrm{O}_{6}$ and separated the phononic and magnetic contributions [20]. The phonon contribution to $1 /\left(T_{1} T\right)$ in $\mathrm{RbOs}_{2} \mathrm{O}_{6}$ does not show a peak, indicating

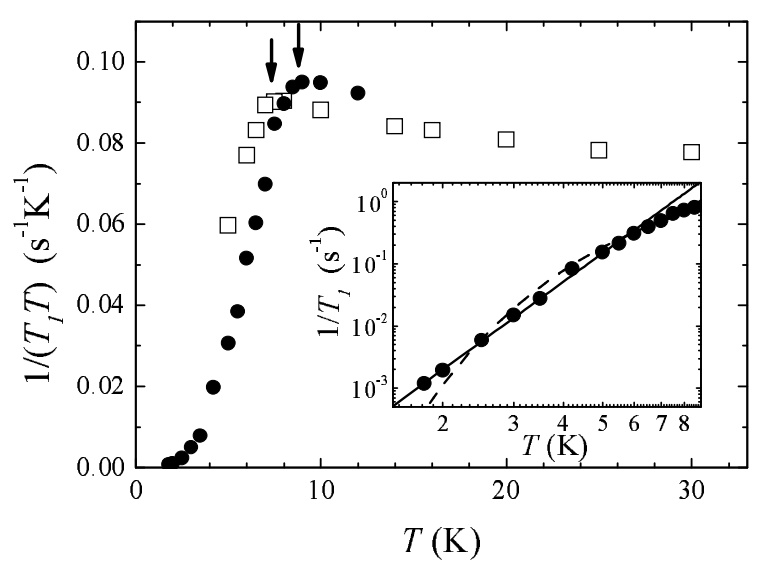

FIG. 3: $T$-dependence of $1 /\left(T_{1} T\right)$ at the ${ }^{17} \mathrm{O}$ sites at $2 \mathrm{~T}$ (solid circles) and $8.5 \mathrm{~T}$ (open squares). The arrows indicate $T_{c}$ at the respective fields. The inset shows the fitting to a powerlaw $1 / T_{1} \propto T^{\alpha}$ with $\alpha=4.7$ (solid line) and an activation law $1 / T_{1} \propto \exp (\Delta / T)$ with $\Delta=17 \mathrm{~K}$ (dashed line) for the data at $2 \mathrm{~T}$ below $T_{c}$. 
underdamped behavior in the whole $T$-range below the room temperature. The $T$-dependence of $\gamma$ in $\mathrm{RbOs}_{2} \mathrm{O}_{6}$ obtained from similar analysis is also compatible with an activation law with $E=12-20 \mathrm{~K}$, although the accuracy is limited.

A remarkable feature of the results in Fig. 2 is the sudden reduction of $\gamma$ below the superconducting $T_{c}$. This indicates that the damping of rattling phonons near $T_{c}$ is primarily caused by electron-phonon interaction and the phonon life time is enhanced by opening of the superconducting gap. Therefore, $\omega_{0}$ should be smaller than $2 \Delta \sim 50 \mathrm{~K}[7]$, where $\Delta$ is the superconducting gap.

We now discuss the results at the $\mathrm{O}$ sites. Figure 3 shows the $T$-dependence of $1 /\left(T_{1} T\right)$ at the $\mathrm{O}$ sites at the fields of $2 \mathrm{~T}$ and $8.5 \mathrm{~T}$. Unlike the result for the $\mathrm{K}$ sites, $1 /\left(T_{1} T\right)$ is only weakly $T$-dependent above $T_{c}$, approximately consistent with the Korringa relation. Thus the spin dynamics of conduction electrons are probed at the $\mathrm{O}$ sites. This is consistent with the Os- $5 d\left(t_{2 q}\right)$ and O- $2 p$ hybridized nature of the conduction band [10, 13]. However, the weak $T$-dependence of $1 /\left(T_{1} T\right)$ in the normal state is still anomalous and suggests development of modest spin correlation.

Below $T_{c}$, it is known that $1 /\left(T_{1} T\right)$ shows a peak near $T=0.9 T_{c}$ (the Hebel-Slichter peak 24] ) in weak coupling superconductors with an isotropic gap. This is not the case for the data in Fig. $3 ; 1 /\left(T_{1} T\right)$ decreases gradually below $T_{c}$, indicating that the HebelSlichter peak is strongly suppressed. At lower temperatures, $T$-dependence of $1 / T_{1}$ can be fit either by a power law $1 / T_{1} \propto T^{\alpha}$ with $\alpha \sim 5$ or by an activation law $1 / T_{1} \propto \exp (-\Delta / T)$ with $\Delta=17 K$. Since the upper critical field $H_{c 2}$ is about $30 \mathrm{~T}$ at $T=0$ [25, 26], pair breaking effects of magnetic field should not be strong at $2 \mathrm{~T}$ [27].

The behavior at low temperatures rules out highly anisotropic gap structure such as line nodes, which leads to $T^{3}$ dependence of $1 / T_{1}$. This is consistent with the field independent thermal conductivity showing an isotropic gap [28]. Therefore, suppression of the HebelSlichter peak cannot be ascribed to a highly anisotropic gap. It appears more likely due to inelastic scattering of quasiparticles by phonons, which broadens the superconducting density of states as was proposed for the case of $\mathrm{TlMo}_{6} \mathrm{Se}_{7.5}$ [29]. This has been also predicted by numerical calculations based on the Eliashberg theory [30, 31]. The calculation by Akis et al. [31], for example, shows that the Hebel-Slichter peak disappears when $k_{B} T_{c} / \hbar \omega_{l n}$ is larger than $0.2 \sim 0.3$, where $\omega_{l n}$ is the Allen-Dynes parameter representing the typical phonon frequency. This is compatible with our previous conclusion that the frequency of the rattling phonon is smaller than $2 \Delta \sim 5 k_{B} T_{c}$. Whether the rattling works as a pairing mechanism or acts as a pair breaker is still an open question.

In conclusion, our analysis of the $1 /\left(T_{1} T\right)$ data at the
$\mathrm{K}$ sites indicates a crossover of rattling from the low $T$ underdamped to high $T$ overdamped behavior near 13 - $16 \mathrm{~K}$. The rapid decrease of $1 /\left(T_{1} T\right)$ at the $\mathrm{K}$ sites below $T_{c}$ indicates strong electron-phonon coupling and enhancement of the lifetime of rattling phonons. Hence its frequency must be smaller than $2 \Delta$. The results at the $\mathrm{O}$ sites rule out strongly anisotropic gap. The absence of the Hebel-Slichter peak at the $\mathrm{O}$ sites is also compatible with strong electron-phonon coupling.

We thank T. Kato, Y. Takada, H. Maebashi K. Ishida, H. Harima, H. Fukuyama, Y. Matsuda, and S. Tajima for valuable discussions. This work was supported by a Grant-in-Aid for Scientific Research on Priority Areas (No. 16076204 "Invention of Anomalous Quantum Materials") from the MEXT Japan.

[1] S. Yonezawa et al., J. Phys.: Condens. Matter 16, L9 (2004).

[2] S. Yonezawa et al., J. Phys. Soc. Jpn. 73, 819 (2004).

[3] M. Brühwiler et al., Phys. Rev. B 70, 020503(R) (2004).

[4] S. Yonezawa, Y. Muraoka, and Z. Hiroi, J. Phys. Soc. Jpn. 73, 1655 (2004).

[5] T. Muramatsu et al., Phys. Rev. Lett. 95, 167004 (2005).

[6] Z. Hiroi et al., J. Phys. Soc. Jpn. 74, 1682 (2005).

[7] Z. Hiroi, S. Yonezawa, Y. Nagao, and J. Yamaura, Phys. Rev. B (to be published).

[8] M. Brühwiler et al., Phys. Rev. B 73, 094518 (2006).

[9] J. Kuněs, T. Jeong, and W. E. Pickett, Phys. Rev. B 70, 174510 (2004).

[10] R. Saniz et al., Phys. Rev. B 70, 100505(R) (2004).

[11] Z. Hiroi et al., J. Phys. Soc. Jpn. 74, 1255 (2005).

[12] J. Yamaura et al., J. Solid State Chem. 179, 336 (2006).

[13] J. Kuneš and W. E. Pickett, Phys. Rev. B 74, 094302 (2006).

[14] V. Keppens et al., Nature 395, 876 (1998).

[15] S. Paschen et al., Phys. Rev. B 64, 214404 (2001).

[16] K. Arai et al., Physica B359-361, 488 (2005).

[17] A. Narath, Phys. Rev. 162, 320 (1967).

[18] A. Abragam, The principles of Nuclear Magnetism (Oxford University Press, Oxford, 1961).

[19] M. Rubinstein and H. A. Resing, Phys. Rev. B 13, 959 (1976).

[20] M. Yoshida et al., unpublished.

[21] B. D. Silverman, Phys. Rev. B 9, 203 (1974).

[22] Y. Takagi, J. Phys. Soc. Jpn. 47, 567 (1979).

[23] J. H. van Vleck and V. F. Weisskopf, Rev. Mod. Phys. 17, 227 (1945).

[24] L. C. Hebel and C. P. Slichter, Phys. Rev. 113, 1504 (1959).

[25] E. Ohmichi et al., J. Phys. Soc. Jpn. 75, 045002 (2006).

[26] T. Shibauchi et al., Phys. Rev. B 74, 220506(R) (2006).

[27] C. H. Pennington and V. A. Strenger, Rev. Mod. Phys. 68, 855 (1996).

[28] Y. Kasahara et al., Phys. Rev. Lett. 96, 247004 (2006).

[29] Y. Kitaoka et al., Physica C192 272 (1992).

[30] P. B. Allen and D. Rainer, Nature 349, 396 (1991).

[31] R. Akis, C. Jiang, and J. P. Carbotte, Physica C176, 485 (1991). 